\title{
FAINT PHOTOMETRY OF EDGE-ON SPIRAL GALAXIES: A SEARCH FOR MASSIVE HALOS
}

\author{
Michael F. Skrutskie, Mark A. Shure, Steven Beckwith \\ Department of Astronomy, Cornell University
}

Upper limits have been set to the luminosity from the massive halos of three late-type edgeon spiral galaxies: NGC 2683 (Sb), NGC 4244 (Scd), and NGC 5907 (Sc). The limits resulted from simultaneous photometry in the visual $(V)$ and $2.2 \mu \mathrm{m}(K)$ photometric bands which is sensitive to both luminosity and color changes along the minor axes of the three galaxies. The $3 \sigma$ lower limits to the mass-to-light ratios for the halo of NGC 5907 are the largest ever recorded: $\mathrm{M} / \mathrm{L}_{V}>2000$ and $\mathrm{M} / \mathrm{L}_{K}>64$ in solar units. Since $K$ band $\mathrm{M} / \mathrm{L}$ for $\mathrm{M}$-dwarf stars lying just above the hydrogen-burning limit is about 35 , the results virtually eliminate the possibility that hydrogen-burning stars comprise more than a fraction of the halo masses. If the halos contain a more realistic spectrum of stellar masses, for example Population II, the visual band measurements imply that these stars account for less than one percent of the halo mass. Similar limits were obtained for NGC 4244 and NGC 2683. Variations of the $V-K$ colors along and perpendicular to the disks show no sign of population changes toward redder objects at large galactocentric radii.

The nucleus of NGC 5907 contains an unresolved source less than 330pc in size with a $2.2 \mu \mathrm{m}$ luminosity of order $5 \times 10^{9} \mathrm{~L}_{\odot}$, and may be an example of a star-burst galactic nucleus overlooked by visual observations. 